ORIGINAL ARTICLE

\title{
Conidial fungi associated with leaf litter of red cedar (Cedrela odorata) in Belém, Pará (eastern Brazilian Amazon)
}

\author{
Renato Ferreira dos SANTOS ${ }^{1}$, Helen Maria Pontes SOTÁO ${ }^{1 *}$, Josiane Santana MONTEIRO ${ }^{1}$, \\ Luís Fernando Pascholati GUSMÃO², Antonio Hernández GUTIÉRREZ ${ }^{3}$ \\ ' Museu Paraense Emílio Goeldi - MPEG, Coordenação de Botânica. Av. Perimetral 1901, Terra firme, Caixa Postal 399, 66077-830 Belém, PA, Brazil. \\ 2 Universidade Estadual de Feira de Santana - UEFS, Departamento de Ciências Biológicas. Avenida Transnordestina, s/n, Novo Horizonte, 44036-900, Feira de \\ Santana, BA, Brazil. \\ 3 Universidade Federal do Pará - UFPA, Instituto de Ciências Biológicas. Rua Augusto Corrêa 01, Caixa Postal 479, 66075-110, Belém, PA, Brazil. \\ * Corresponding author: helen@museu-goeldi.br
}

\section{ABSTRACT}

The aim of this study was to investigate the species of conidial fungi associated with leaf litter of Cedrela odorata (Meliaceae), an endangered red cedar species typical of Amazonian terra-firme forests. Conidial fungi were sampled around C. odorata individuals in three forest areas in the municipality of Belém (Pará State, Brazil). A total of 104 species were identified, with 53 new records for the state of Pará, 46 first records for the Brazilian Amazon, including new records for Brazil (Cordana abramovii), for South America (Acarocybiopsis cubitaensis, Xylocladium claviforme) and for the Americas (Dactylaria biguttulata). A review of species of conidial fungi reported on C. odorata is provided, indicating its distribution in Brazil. For each new record in Brazil, South America and the Americas we present a description, illustrations, geographical distribution and taxonomic comments.

KEYWORDS: Ascomycota, hyphomycetes, Meliaceae, tropical forest

\section{Fungos conidiais associados com o folhedo de cedro vermelho (Cedrela odorata) em Belém, Pará (Amazônia Oriental brasileira)}

\section{RESUMO}

O objetivo deste estudo foi investigar as espécies de fungos conidiais associadas com o folhedo de Cedrela odorata, uma espécie de cedro vermelho ameaçada, típica de florestas de terra firme da Amazônia. Fungos conidiais foram amostrados no folhedo ao redor de indivíduos de $C$. odorata em três áreas florestais no município de Belém (PA). Um total de 104 espécies foi identificado, com 53 novos registros para o Estado do Pará e 46 que representam o primeiro registro para a Amazônia brasileira. Dentre estas, foram encontrados novos registros para o Brasil (Cordana abramovii), para a América do Sul (Acarocybiopsis cubitaensis, Xylocladium claviforme) e para as Américas (Dactylaria biguttulata). Uma revisão das espécies de fungos conidiais relatadas em C. odorata é fornecida, indicando sua distribuição no Brasil. Para cada novo registro no Brasil, América do Sul e Américas apresentamos uma descrição, ilustrações, distribuição geográfica e comentários taxonômicos.

PALAVRAS-CHAVE: Ascomycota, hyphomycetes, Meliaceae, floresta tropical

\section{INTRODUCTION}

Conidial fungi are active components in the process of plant decomposition (Dix and Webster 1995). Several studies in Brazil have surveyed the species richness and diversity of these fungi in association with plant debris of several botanical families, revealing a significant number of new species and records for the country (e.g. Forzza et al. 2010; Flora do Brasil $2020 \mathrm{em}$ construção 2017; CRIA 2017).
The pioneering studies on conidial fungi in the Brazilian Amazon were by Hennings (1900) and Batista et al. (1966). Later contributions increased the knowledge and richness of these organisms in upland and lowland forests (Hernández-Gutiérrez et al. 2009; Monteiro et al. 2010; Castro et al. 2012; Carmo et al. 2014).

Cedrela odorata L. (Meliaceae), known as red cedar, is an Amazonian timber species of great economic interest, exploited for the production of noble woods of high commercial value, and is currently threatened by extinction (Albernaz and Avila- 
Pires 2009; IUCN 2017). The distribution of this species in Brazil covers areas of the Amazon, Cerrado and Atlantic Forest biomes (Forzza et al. 2010).

Only a few studies have reported on the fungal species associated with Cedrela in Brazil, including some conidial fungi and sexual Ascomycetes. Hennings (1902), Gusmão and Grandi (1997) and Viana et al. (2012) reported fungi on C. fissilis Vell. in the states of São Paulo, Paraná and Ceará, respectively. Batista et al. (1964) and Hanada et al. (2005) reported Phyllachora balansae Speg. and Pseudobeltrania cedrelae Henn., respectively, to cause leaf spot in $C$. odorata in the state of Amazonas.

Considering the lack of information on the occurrence of fungi associated with plant species on the verge of extinction in Brazil, and the current conservation status of this tree species of great commercial value, our objective was to study the conidial fungi associated with $C$. odorata in three forest remnants in the highly impacted Belém endemism center, in the eastern Brazilian Amazon. Our aim was to survey the diversity of conidial fungi associated with the leaf litter of $C$. odorata, and to describe and illustrate new records for Brazil.

\section{MATERIAL AND METHODS}

We selected three areas in the municipality of Belém (state of Pará, northern Brazil) because they contained $C$. odorata specimens: (a) Bosque Rodrigues Alves Botanical Garden (BRA) (1'25'47"S, $48^{\circ} 27^{\prime} 16^{\prime \prime} \mathrm{W}$ ), an area of upland forest (Ferreira et al. 2012); (b) Zoobotanical Park of the Museu Paraense Emílio Goeldi (ZPM) (1 $\left.1^{\circ} 27^{\prime} 14^{\prime \prime} \mathrm{S}, 48^{\circ} 28^{\prime} 31^{\prime \prime} \mathrm{W}\right)$, also an upland forest fragment (Ferreira et al. 2012); and (c) Environmental Conservation Area (APA) of Combu Island (1030'40"S, 48027'35"W), which contains lowland, seasonally flooded forest (Castro et al. 2012).

Five individuals of $C$. odorata were selected in each area, and six bimonthly collections were carried out between December 2014 and October 2015. The trees were chosen considering the amount of litter available at their bases. During each collection, litter samples were collected from each individual, consisting of leaflets, rachis, branches and fruits (when available) in the process of decomposition.

Fungi were accessed using an adaptation of the technique of Castañeda-Ruiz et al. (2016). Samples were washed in tap water and then incubated in adapted wet chambers consisting of $1 \mathrm{~L}$ plastic bags lined with moistened paper towel at the bottom. The wet chambers were placed in a polystyrene box $(80 \mathrm{~L})$ with $500 \mathrm{ml}$ of water $+2 \mathrm{ml}$ of glycerin and lined with moistened towel paper. Five times a week the styrofoam box was opened for 30 minutes for air circulation. The moisture in the box was maintained by spraying water on the paper towel when needed.

After 72 hours, the samples were analyzed under a stereomicroscope to verify reproductive structures. The examination was repeated for a period of $30-45$ days. When present, the structures were removed from the substrate using thin needles and mounted on semi-permanent slides with lactoglycerol (distilled water + lactic acid + glycerin) (Carmo et al. 2016) and permanent slides with PVL resin (polyvinyl alcohol + lactophenol) (Trappe and Schenck 1982). Species identification was achieved through morphological analysis and measurement of the microstructures of taxonomic value under an optical microscope with the aid of specialized literature, such as Ellis (1971), Matsushima (1975) and Seifert et al. (2011). Illustrations were prepared based on photomicrographs obtained from a DP25 digital camera coupled to an Olympus BX51 optical microscope, equipped with or without a differential interference contrast (DIC) prism.

For each identified species the geographic distribution in Brazil was determined based on CRIA (2017), and for each new species in Brazil a morphological description, illustrations and taxonomic comments are presented. The samples and slides were deposited in the Herbarium João Murça Pires (MG) of the Museu Paraense Emílio Goeldi (MPEG).

The collection of specimens at the Zoobotanical Park, APA of Combu Island and Bosque Rodrigues Alves was authorized by Instituto Chico Mendes de Conservação da Biodiversidade (SISBIO permit \#11937) and Secretaria do Municipal do Meio Ambiente - SEMMA/Belém (permit dates 10/12/2014 and 10/07/2015).

\section{RESULTS}

We identified 104 species of conidial fungi associated with the litter of $C$. odorata (Table 1). Among these, 53 new records for the state of Pará were found, of which 46 represented new records for the Brazilian Amazon (Table 1). Cordana abramovii Seman \& Davydkina is a new record for Brazil, while Acarocybiopsis cubitaensis J. Mena, A. Hern. Gut. \& Mercado and Xylocladium claviforme (J.L. Crane \& Dumont) Arx are new records for South America. Dactylaria biguttulata Goh \& K.D. Hyde was registered for the first time in the Americas.

\section{Acarocybiopsis cubitaensis J. Mena, A. Hern. Gut. \& Mercado, Mycological Research 103 (8): 1032 (1999)}

Figure $1 \mathrm{~A}-\mathrm{C}$

Synnemata solitary, straight or slightly flexuous, brown to dark brown, 42.5-125 × 10-12.5 $\mu \mathrm{m}$; composed by an ascending hypha that supports a single conidiogenous cell and descending hyphae formed in the basal part of each conidiogenous cell. Hyphae septate, smooth, brown to dark brown. Conidiogenous cells monoblastic, terminal, discrete, lageniform, with 3-5 percurrent extensions. Conidia acrogenous, solitary, ellipsoid to obovoid, 3-septate, rounded at the apex, median septum with a black band, not constricted at the septa, smooth, brown to dark brown, $25-35 \times 22.5-25 \mu \mathrm{m}$.

Material examined: Brazil. Pará: Belém, Parque Zoobotânico do Museu Paraense Emílio Goeldi, on decaying wood of Cedrela odorata, 16/X/2015, R.F. Santos 100 (MG 226161). 
Table 1. List of 104 species of conidial fungi recorded on the leaf litter of Cedrela odorata in the metropolitan area of Belém (state of Pará, Brazil). Distribution in Brazil indicates the states in which the species was recorded, and is based on CRIA (2017) and data of this study (in bold). New records: *American continent; ${ }^{\bullet}$ South America; -Brazil; ‘ Brazilian Amazon; *Pará state. APA - Environmental Conservation Area of Combu Island; BRA - Bosque Rodrigues Alves Botanical Garden; ZPM - Zoobotanical Park of the Museu Paraense Emílio Goeldi.

\begin{tabular}{|c|c|c|c|c|}
\hline Species & APA & BRA & ZPM & Distribution in Brazil \\
\hline Acarocybiopsis cubitaensis J. Mena, A. Hern. Gut. \& Mercado.*A * & & & $x$ & PA \\
\hline Acrogenospora sphaerocephala (Berk. \& Broome) M.B. Ellis & $x$ & $x$ & $x$ & $B A, C E, P A$ \\
\hline Acumispora verruculosa Heredia, R.F. Castañeda \& R.M. Arias & $x$ & $x$ & & $\mathrm{PA}, \mathrm{SP}$ \\
\hline Anungitea fragilis B. Sutton * & & $x$ & & $B A, \mathbf{P A}$ \\
\hline Arthrinium sacchari (Speg.) M.B. Ellis`* & $x$ & $x$ & & $\mathbf{P A}, P E$ \\
\hline Beltrania rhombica Penz. & $x$ & $x$ & $x$ & $A M, B A, C E, P A, P B, P E, P I, P R, R N, S P, T O$ \\
\hline Beltraniella portoricensis (F. Stevens) Piroz. \& S.D. Patil & $x$ & $x$ & $x$ & $A M, B A, C E, P A, P B, P E, P I, P R, R N, S P$ \\
\hline Beltraniopsis esenbeckiae Bat. \& J.L. Bezerra॥ * & & $x$ & $x$ & $B A, \mathbf{P A}, P B, P E$ \\
\hline Berkleasmium corticola (P. Karst.) R.T. Moore ${ }^{\mathbf{\Delta}}$ & & & $x$ & $B A, \mathbf{P A}$ \\
\hline Brachiosphaera tropicalis Nawawi & $x$ & & & BA, PA \\
\hline Brachysporiella gayana Bat. & & & $x$ & $A M, B A, C E, P A, P E, P I, S P$ \\
\hline Cacumisporium pleuroconidiophorum (Davydkina\&Melnik) R.F. Castañeda, Heredia \& Iturr. & & $x$ & $x$ & $B A, P A$ \\
\hline Camposporium fusisporum Whitton, McKenzie \& K.D. Hyde & & & $x$ & PA \\
\hline Campylospora filicladia Nawawi^ * & $x$ & & & $C E, \mathbf{P A}$ \\
\hline Canalisporium caribense (Hol.-Jech. \& Mercado) Nawawi \& Kuthub. & $x$ & $x$ & $x$ & $B A, P A$ \\
\hline Canalisporium elegans Nawawi \& Kuthub. & & $x$ & & PA \\
\hline Canalisporium pulchrum (Hol.-Jech. \& Mercado) Nawawi \& Kuthub. & $x$ & $x$ & $x$ & PA \\
\hline Cancellidium applanatum Tubaki & $x$ & & & $\mathrm{AM}, \mathrm{BA}, \mathrm{PA}$ \\
\hline Candelabrum brocchiatum Tubaki & & & $x$ & $B A, P A, R N, S P$ \\
\hline Chaetendophragmia triangularis Matsush. & $x$ & & & $B A, P A, S P$ \\
\hline Chlamydopsis proliferans Hol.-Jech. \& R.F. Castañeda ${ }^{*}$ & & & $x$ & $\mathrm{BA}, \mathbf{P A}, \mathrm{SP}$ \\
\hline Chloridium reniforme Matsush.. ${ }^{*}$ & & $x$ & & PA \\
\hline Ch. virescens (Pers.) W. Gams \& Hol.-Jech.* & & & $x$ & $A M, B A, C E, \mathbf{P A}, S P$ \\
\hline Circinotrichum britannicum P.M. Kirk`* & & $x$ & & $B A, C E, \mathbf{P A}$ \\
\hline Circinotrichum maculiforme Nees ${ }^{\mathbf{\Delta}}$ & $x$ & $x$ & $x$ & $\mathrm{BA}, \mathbf{P A}, \mathrm{SP}$ \\
\hline Circinotrichum olivaceum (Speg.) Piroz.. * & & & $x$ & $B A, C E, \mathbf{P A}, P B, R N, S P$ \\
\hline Circinotrichum papakuraeS. Hughes \&Piroz. ${ }^{*}$ & $x$ & & & $\mathrm{BA}, \mathrm{CE}, \mathbf{P A}, \mathrm{PI}, \mathrm{SP}$ \\
\hline Clonostachys rosea (Link) Schroers, Samuels, Seifert \& W. Gams & & $x$ & & $B A, P A, P B, P E, R N, S P$ \\
\hline Codinaea assamica (Agnihothr.) S. Hughes \& W.B. Kendr. & $x$ & $x$ & $x$ & BA, PA, RN \\
\hline Codinaea fertilis S. Hughes \& W.B. Kendr. & $x$ & $x$ & $x$ & $B A, C E, P A, P E, P B, P I, S P$ \\
\hline Codinaea simplex S. Hughes \& W.B. Kendr. & $x$ & $x$ & & $A M, B A, C E, P A, P B, P E, P I, S P$ \\
\hline Coleodictyospora micronesiaca (Matsush.) Matsush. & $x$ & & & PA \\
\hline Conioscypha lignicola Höhn.凶* & & $x$ & & PA \\
\hline Conioscypha varia Shearer & & & $x$ & BA, PA, RN \\
\hline Cordana abramovii Seman \& Davydkina"A * & $x$ & & & PA \\
\hline Cryptophiale kakombensis Piroz. & & $x$ & & $\mathrm{AM}, \mathrm{BA}, \mathrm{CE}, \mathrm{PA}, \mathrm{PE}, \mathrm{PI}, \mathrm{SP}$ \\
\hline Cryptophiale udagawae Piroz. \& Ichinoe & & $x$ & $x$ & $A P, B A, C E, P A, P B, P I, R N, S P$ \\
\hline Cylindrocladium naviculatum Crous\& M.J. Wingf.^* & & & $x$ & $B A, \mathbf{P A}$ \\
\hline Dactylaria biguttulata Goh \& K.D. Hyde...A * & $x$ & $x$ & & PA \\
\hline Dactylaria candidula (Höhn.) G.C. Bhatt \& W.B. Kendr. & $x$ & & & $A M, B A, C E, P A, R N, S P$ \\
\hline Dendryphiopsis atra (Corda) S. Hughes ${ }^{\star} *$ & & & $x$ & $\mathrm{BA}, \mathbf{P A}, \mathrm{RN}$ \\
\hline Dictyochaetopsis polysetosa R.F. Castañeda, Gusmão, Guarro \& Saikawa & & $x$ & & $B A, P A, R J$ \\
\hline Dictyosporium digitatum J.L. Chen, C.H. Hwang \& Tzean & & $x$ & & $A P, B A, P A$ \\
\hline Dictyosporium elegans Corda ${ }^{*}$ & $x$ & $x$ & $x$ & $\mathrm{BA}, \mathrm{CE}, \mathbf{P A}$ \\
\hline Dictyosporium musae Photita & $x$ & & $x$ & PA \\
\hline
\end{tabular}


Table 1. Continued

\begin{tabular}{|c|c|c|c|c|}
\hline Species & APA & BRA & ZPM & Distribution in Brazil \\
\hline Dinemasporium lanatum Nag Raj \& R.F. Castañeda & $x$ & $x$ & & $\mathrm{AM}, \mathrm{BA}, \mathrm{PA}, \mathrm{RN}$ \\
\hline Diplocladiella scalaroides G. Arnaud & & $x$ & $x$ & $B A, P A$ \\
\hline Ellisembia adscendens (Berk.) Subram. & $x$ & $x$ & $x$ & $\mathrm{BA}, \mathrm{CE}, \mathrm{PA}, \mathrm{PB}, \mathrm{PE}, \mathrm{PI}, \mathrm{RN}, \mathrm{SP}$ \\
\hline Ellisembia bambusae (M.B. Ellis) W.P. Wu ${ }^{*}{ }^{*}$ & & & $x$ & $B A, \mathbf{P A}$ \\
\hline Ellisembia minigelatinosa (Matsush.) W.P. Wu & & $x$ & & $B A, P A, R N$ \\
\hline Ellisembia vaga (Nees \& T. Nees) Subram. ${ }^{*}{ }^{*}$ & $x$ & $x$ & $x$ & $\mathrm{BA}, \mathbf{P A}, \mathrm{RN}$ \\
\hline Exserticlava triseptata (Matsush.) S. Hughes & $x$ & $x$ & & $A M, B A, P A, P B$ \\
\hline Gonytrichum macrocladum (Sacc.) S. Hughes ${ }^{\mathbf{*}}$ & $x$ & $x$ & $x$ & $\mathrm{BA}, \mathbf{P A}, \mathrm{PE}, \mathrm{SP}$ \\
\hline Gyrothrix circinata (Berk. \& M.A. Curtis) S. Hughes ${ }^{\star} *$ & & $x$ & $x$ & $\mathrm{BA}, \mathrm{CE}, \mathbf{P A}, \mathrm{PE}, \mathrm{PR}, \mathrm{RN}, \mathrm{SP}$ \\
\hline Gyrothrix podosperma (Corda) Rabenh..* & $x$ & $x$ & $x$ & $\mathrm{BA}, \mathrm{CE}, \mathbf{P A}, \mathrm{PR}, \mathrm{SP}$ \\
\hline Hansfordia pulvinata (Berk. \& M.A. Curtis) S. Hughes* & $x$ & & & $A M, B A, \mathbf{P A}$ \\
\hline Helicomyces roseus Link & $x$ & $x$ & & $B A, P A, P B, R S$ \\
\hline Helicosporium aureum (Corda) Linder ${ }^{\mathbf{\Delta}}$ & & $x$ & $x$ & $B A, C E, \mathbf{P A}$ \\
\hline Helicosporium pannosum (Berk. \& M.A. Curtis) R.T. Moore & $x$ & & & $B A, P A, S P$ \\
\hline Helicosporium griseum Berk. \& M.A. Curtis ${ }^{*}$ & & & $x$ & $\mathrm{BA}, \mathbf{P A}, \mathrm{PE}, \mathrm{SP}$ \\
\hline Helicosporium guianense Linder ${ }^{\star} *$ & $x$ & & $x$ & $B A, \mathbf{P A}$ \\
\hline Helicosporium virescens (Pers.) Sivan. & & & $x$ & $B A, P A, P E, R N$ \\
\hline Hermatomyces sphaericus (Sacc.) S. Hughes ${ }^{\mathbf{*}}$ & $x$ & $x$ & $x$ & $B A, \mathbf{P A}$ \\
\hline Idriella cubensis R.F. Castañeda \& G.R.W. Arnold ${ }^{\mathbf{\Delta}}$ * & $x$ & $x$ & $x$ & PA \\
\hline Idriella lunata P.E. Nelson \& S. Wilh. ${ }^{*}$ * & $x$ & & $x$ & $\mathrm{BA}, \mathbf{P A}, \mathrm{PE}, \mathrm{PI}$ \\
\hline Junewangia globulosa (Tóth) W.A. Baker \& Morgan-Jones ${ }^{*}$ * & $x$ & & & $B A, P A, P B, P E, R N$ \\
\hline Lauriomyces sakaeratensis Somrith., Kosol \& E.B.G. Jones & & $x$ & & $\mathrm{AM}, \mathrm{BA}, \mathrm{PA}$ \\
\hline Mariannaea elegans (Corda) Samson & & $x$ & & $\mathrm{BA}, \mathrm{PA}, \mathrm{SP}$ \\
\hline Megacapitula villosa J.L. Chen \&Tzean«* & & & $x$ & PA \\
\hline Melanocephala triseptata (Shearer, J.L. Crane \& M.A. Mill.) S. Hughes ${ }^{\mathbf{*}}$ & $x$ & $x$ & $x$ & $\mathrm{BA}, \mathbf{P A}$ \\
\hline Menisporopsis pirozynskii Varghese \& V.G. Rao & & & $x$ & $A P, B A, P A, R N, S P$ \\
\hline Menisporopsis theobromae S. Hughes & $x$ & $x$ & $x$ & $A P, B A, C E, P A, P B, P E, P I, R N, S P$ \\
\hline Monodictys paradoxa (Corda) S. Hughes & $x$ & $x$ & $x$ & PA \\
\hline Monotosporella palmicola Yanna \& K.D. Hyde & $x$ & & & PA \\
\hline Myrothecium setiramosum R.F. Castañeda $\mathbf{\Lambda}$ & $x$ & $x$ & & $B A, \mathbf{P A}$ \\
\hline Nigrospora sphaerica (Sacc.) E.W. Mason* & $x$ & & $x$ & $A M, A L, P A, P E, R J, S P$ \\
\hline Paliphora intermedia Alcorn & & $x$ & & $\mathrm{AM}, \mathrm{BA}, \mathrm{PA}, \mathrm{PI}$ \\
\hline Periconia cookie E.W. Mason \& M.B. Ellis^* & & $x$ & & $\mathrm{BA}, \mathrm{CE}, \mathrm{GO}, \mathrm{MA}, \mathbf{P A}, \mathrm{PE}, \mathrm{PI}, \mathrm{RN}, \mathrm{SP}$ \\
\hline Peyronelina glomerulata P.J. Fisher, J. Webster \& D.F. Kane ${ }^{\mathbf{\Delta}}$ & & & $x$ & $\mathbf{P A}, P E$ \\
\hline Phaeocandelabrum elegans (R.F. Castañeda) R.F. Castañeda, Heredia \& Saikawa * & & & $x$ & $B A, C E, \mathbf{P A}$ \\
\hline Phaeoisaria clematidis (Fuckel) S. Hughes & $x$ & $x$ & $x$ & $B A, C E, P A, P E, R N, S P$ \\
\hline Phaeoisaria triseptata Hol.-Jech. ${ }^{*}$ & & $x$ & & $B A, C E, \mathbf{P A}$ \\
\hline Physalidiella elegans (LuppiMosca) Rulamort & $x$ & & & $B A, P A, S P$ \\
\hline Piricauda cochinensis (Subram.) M.B. Ellis & $x$ & & & $B A, P A, P B$ \\
\hline Pithomyces chartarum (Berk. \& M.A. Curtis) M.B. Ellis* & & & $x$ & $\mathrm{AM}, \mathrm{BA}, \mathbf{P A}, \mathrm{PE}, \mathrm{PI}, \mathrm{RN}, \mathrm{SP}$ \\
\hline Polyschema amoenum R.F. Castañeda, Iturr. \& Minter & & & $x$ & PA \\
\hline Repetophragma fasciatum (R.F. Castañeda) R.F. Castañeda, Gusmão \& Saikawa॥ * & $x$ & $x$ & & $\mathrm{BA}, \mathbf{P A}$ \\
\hline Repetophragma filiferum (Piroz.) R.F. Castañeda, Gusmão \& Heredia & $x$ & & $x$ & $P A, P E, S P$ \\
\hline Rhexoacrodictys erecta (Ellis \&Everh.) W.A. Baker \& Morgan-Jones & $x$ & & & $B A, P A, P B, R N, S P$ \\
\hline Solosympodiella clavata Matsush. ${ }^{*}$ & & $x$ & & $B A, \mathbf{P A}$ \\
\hline Speiropsis pedatospora Tubaki * & $x$ & & & $\mathrm{BA}, \mathbf{P A}$ \\
\hline Speiropsis scopiformis Kuthub. \& Nawawi & $x$ & $x$ & & $A P, B A, C E, P A, P I, S P$ \\
\hline
\end{tabular}


Table 1. Continued

\begin{tabular}{|c|c|c|c|c|}
\hline Species & APA & BRA & ZPM & Distribution in Brazil \\
\hline Sporidesmium tropicale M.B. Ellis ${ }^{\star}$ & $x$ & $\mathrm{x}$ & $\mathrm{x}$ & $\mathrm{BA}, \mathrm{CE}, \mathbf{P A}, \mathrm{PB}$ \\
\hline Stachybotrys kampalensis Hansf. ${ }^{*}$ & & $x$ & & $B A, \mathbf{P A}$ \\
\hline Stachybotrys longispora Matsush. ${ }^{*}$ & & & $x$ & $B A, C E, \mathbf{P A}$ \\
\hline Tetraploa aristata Berk. \& Broome & $x$ & & & $B A, P A, P E, R N, S P$ \\
\hline Thozetella cristata Piroz. \& Hodges & $x$ & $x$ & & $\mathrm{BA}, \mathrm{CE}, \mathrm{PA}, \mathrm{PE}, \mathrm{PI}, \mathrm{PR}, \mathrm{RN}, \mathrm{SP}$ \\
\hline Triadelphia uniseptata (Berk. \& Broome) P.M. Kirk * & & $x$ & & PA, RS \\
\hline Vanakripa fasciata R.F. Castañeda, M. Stadler \& Decock & & $x$ & & PA \\
\hline Vermiculariopsiella cubensis (R.F. Castañeda) Nawawi, Kuthub. \& B. Sutton * & & $x$ & & $\mathrm{BA}, \mathbf{P A}, \mathrm{SP}$ \\
\hline Wiesneriomyces laurinus (Tassi) P.M. Kirk & $x$ & $x$ & $x$ & $B A, C E, P A, P B, P E, P I, P R, R N, S P$ \\
\hline Xylocladium claviforme (J.L. Crane \& Dumont) Arx.“A * & & & $x$ & PA \\
\hline Zygosporium echinosporum Bunting \& E.W. Mason* & $x$ & & & $A P, B A, P A, P E$ \\
\hline Zygosporium gibbum (Sacc., M. Rousseau \& E. Bommer) S. Hughes* & $x$ & & & $\mathrm{AM}, \mathrm{BA}, \mathrm{CE}, \mathbf{P A}, \mathrm{PE}, \mathrm{Pl}, \mathrm{RN}$ \\
\hline Zygosporium masonii S. Hughes* & $x$ & $x$ & $x$ & $A P, A M, B A, P A, P E, R N, S P$ \\
\hline Total $=104$ & 58 & 59 & 56 & \\
\hline
\end{tabular}

Known distribution: America [Brazil (this study), Cuba (Mena-Portales et al. 1999)].

Comments: Acarocybiopsis cubitaensis was proposed by Mena-Portales et al. (1999) and remains the single species of a monotypic genus; it is characterized by a synnema consisting of ascending hyphae that support the conidiogenic cell from which descending hyphae originate, which extend towards the substrate. Conidiogenesis is monoblastic and the conidiogenous cells have percurrent extensions producing ellipsoid to obovoid, 3-septate, brown conidia. Seifert et al. (2011) suggested that Manoharachariomyces lignicola N.K. Rao, D.K. Agarwal \& Kunwar (Rao et al. 2005) would be congeneric with Acarocybiopsis, but would represent a different species because it has 2-3, strongly interlaced hyphae arising from the base of the conidiogenous cell, forming a synnema. The hyphae and conidia of M. lignicola are smaller (up to 110 $\times 3.5-4.5 \mu \mathrm{m}$ and $28-36 \times 16-18.5 \mu \mathrm{m}$ respectively) than those found in A. cubitaensis. A careful analysis of the $M$. lignicola holotype is necessary for a new combination to be proposed. The morphological characteristics of the specimens studied are in agreement with the description of the holotype (Mena-Portales et al. 1999), however the measurements found in the Brazilian material were smaller than those described for the synnemata $(42.5-125 \times 10-12.5 \mu \mathrm{m}$ vs. $50-300 \times$ $12-20 \mu \mathrm{m})$ and conidia [25-35 $\times 22.5-25 \mu \mathrm{m}$ vs. $(35.5-)$ $38-46 \times(18.5) 21.5-26 \mu \mathrm{m}]$. It should be noted that this is the first time that $A$. cubitaensis has been collected outside the type locality. This represents the first record of this species for South America.

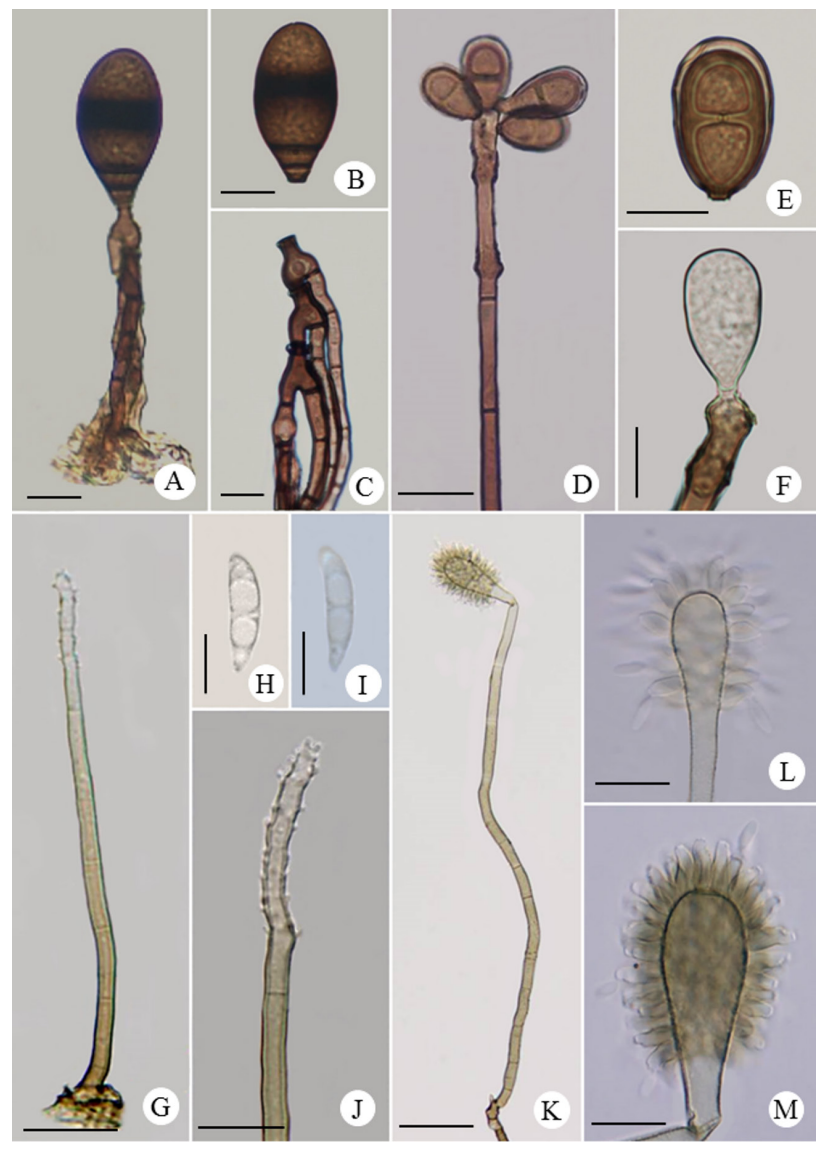

Figure 1. A-C. Acarocybiopsis cubitaensis: A. Synnema, B. Conidium, C. Detail of conidiogenous cells; D-F. Cordana abramovii: D. Conidiophore and conidia, E. Conidium, F. Conidiogenous cell and young conidium; G-J. Dactylaria biguttulata: G. Conidiophore, H-I. Conidium, J. Detail of conidiogenous cell; K-M. Xylocladium claviforme: K. Conidiophore, L. Vesicle and conidia, M. Detail of vesicle. Bars: $10 \mu \mathrm{m}(\mathrm{A}-\mathrm{C}, \mathrm{E}-\mathrm{F}, \mathrm{H}-\mathrm{J}, \mathrm{L}-\mathrm{M}), 20 \mu \mathrm{m}(\mathrm{D}, \mathrm{G}), 50 \mu \mathrm{m}(\mathrm{K})$. This figure is in color in the electronic version. 


\section{Cordana abramovii Seman \& Davydkina, Novosti Sistematiki Nizshikh Rastenii 20: 115 (1983)}

Figure 1D-F

Conidiophores macronematous, mononematous, simple, erect, straight or slightly flexuous, septate, thick walled, smooth, dark brown, paler towards the apex, 225-325 × 7.5$8.5 \mu \mathrm{m}$. Conidiogenous cells polyblastic, integrated, terminal and intercalary, usually swelling, 7.5-10 $\mu \mathrm{m}$ wide, denticulate. Conidia acropleurogenous, solitary, pyriform to obovoid, 1-distoseptate, with a septal pore, thick walled, smooth, brown to dark brown, 20-30 × 15-17.5 $\mu \mathrm{m}$, truncated base.

Material examined: Brazil. Pará: Belém, Combu Island, on decaying wood of Cedrela odorata, 18/X/2014, R.F. Santos 89 (MG 226165).

Known distribution: Africa [Seychelles (Zelski et al. 2014)], America [Brazil (this study), Costa Rica, Nicaragua (Delgado and Koukol 2016),Mexico (Heredia-Abarca et al. 2006), Peru (Zelski et al. 2014), USA (Raja et al. 2007)], Asia [Brunei, Philippines (Cai et al. 2003), India (Heredia-Abarca et al. 2006), Russia, Thailand (Zelski et al. 2014)], Oceania [New Zealand (Heredia-Abarca et al. 2006)].

Comments: Cordana was described by Preuss (1851) and typified by C. pauciseptata Preuss. This genus has simple, macronematous conidiophores with polyblastic, sympodial conidiogenous cells that are terminal or intercalary, usually inflated, with cylindrical denticles that form 0 -1-septate, brown to light brown conidia, with schizolytic secession (Seifert et al. 2011; Hernández-Restrepo et al. 2014). Currently, 19 species of Cordana are included in Cordanaceae (Cordanales, Sordariomycetes), which can be found as saprobes or phytopathogens (Hernández-Restrepo et al. 2014; Zelski et al. 2014). Most of these species are known only from the type locality, with the exception of C. abramovii, C. musae (Zimm.) Höhn. and C. terrestris (Timonin) M. Hern.-Rest., Gené \& Guarro, which present wide distributions (Farr and Rossman 2017). In Brazil, C. ellipsoidea de Hoog (Bahia, Pará), C. musae (Bahia, Pará, Piauí), C. pauciseptata (Bahia) and C. terrestris (Bahia) were recorded (CRIA 2017). Cordana abramovii was found in basidiomata of a Polyporales and in decomposing wood of Beilschmiedia tarairi (A. Cunn.) Kirk, (Lauraceae), with conidiophores measuring up to $1000 \mu \mathrm{m}$ and conidia of 27-31 $\times 15-15.5 \mu \mathrm{m}$ (Seman and Davydkina 1983). Morphologically, the Brazilian specimens are consistent with the description of the holotype, except for the smaller conidiophores (225-325 vs. $250-1000 \mu \mathrm{m})$. This species is widely distributed in temperate and tropical areas, this being the first record for Brazil.

\section{Dactylaria biguttulata Goh \& K.D. Hyde, Fungal Diversity 3: 64 (1999)}

Figure 1G-J

Conidiophores macronematous, mononematous, simple, erect, straight or slightly flexuous, septate, smooth, brown, paler towards the apex, $92.5-137.5 \times 3-3.5 \mu \mathrm{m}$. Conidiogenous cells polyblastic, integrated, terminal, denticulate, subhyaline to very pale brown. Conidia acropleurogenous, ellipsoid, 1-septate, slightly curved, obtuse to subobtuse at the apex, with a guttule in each cell, smooth, hyaline, 10-12 × 3-4 $\mu \mathrm{m}$.

Material examined: Brazil. Pará: Belém, Jardim Botânico Bosque Rodrigues Alves, on decaying fruit of $C$. odorata, 28/ IV/2015, R.F. Santos 32 (MG 226166); Combu Island, on decaying wood of C. odorata, 10/VI/2015, R.F. Santos 49 (MG 226667).

Known distribution: America [Brazil (this study)], Asia [China (Goh and Hyde 1999)].

Comments: Dactylaria was proposed by Saccardo (1880) with D. purpurella (Sacc.) Sacc. as the type species. This genus is characterized by simple or rarely branched, hyaline or brown conidiophores with polyblastic, sympodial, denticulate conidiogenous cells, with unicellular to multiseptate, hyaline or brown conidia with schizolytic secession (Seifert et al. 2011). Worldwide, 87 species are currently accepted, distributed in temperate and tropical areas (Goh and Hyde 1997; Paulus et al. 2003; Mycobank 2017). In Brazil, 20 species have been recorded in the Amazon, Caatinga and Atlantic Forest biomes, with D. candidula (Höhn.) G.C. Bhatt \& W.B. Kendr. the most common species (CRIA 2017). Dactylaria biguttulata was described by Goh and Hyde (1999) on submerged wood. According to Goh and Hyde (1999) the conidia of $D$. biguttulata can be compared to those of D. longidentata Cazau, Aramb. \& Cabello in terms of the presence of guttule and conidial morphology. However, the conidia of $D$. longidentata are predominantly unicellular and smaller $(8-11 \times 2-3$ vs. $10-13 \times 3.5-4.5 \mu \mathrm{m})$ (Cazau et al. 1990). This is the first report of the species for the terrestrial environment and for the Americas.

\section{Xylocladium claviforme (J.L. Crane \& Dumont) Arx, Proceedings van de Koninklijke Nederlandse Akademie van Wetenschappen Section C 85: 27 (1982)}

Figure 1K-M

Conidiophores macronematous, mononematous, simple or branched, straight or slightly flexuous, septate, verrucose, brown, paler towards the apex, 320-600 × 8-10 $\mu$ m, apex enlarged forming a vesicle; clavate vesicle, pale brown, $25-35 \times 12-15 \mu \mathrm{m}$. Conidiogenous cells polyblastic, discrete, sympodial, ellipsoid, pale brown, cicatrized, formed in the vesicle. Secession rhexolytic. Conidia acrogenous, solitary, unicellular, fusiform to ellipsoid, obtuse at the apex, truncated at the base, smooth, subhyaline, $6-8 \times 2.5-3 \mu \mathrm{m}$.

Material examined: Brazil. Pará: Belém, Parque Zoobotânico do Museu Paraense Emílio Goeldi, on decaying wood of Cedrela odorata, 10/VI/2015, R.F. Santos 77 (MG 226170). 
Known distribution: America [Brazil (this study), Cuba, Mexico (Heredia et al. 2004), Jamaica (Crane and Dumont 1975)].

Comments: Xylocladium was proposed by Engler and Prantl (1900) and typified by X. clautriavii (Pat.) P. Syd. ex Lindau. This genus is included in the Graphostromataceae (Xylariales) and is characterized by the presence of macronematous conidiophores, usually forming a vesicle clavate at the apex, where cicatrized, sympodial conidiogenous cells produce unicellular, hyaline conidia with rhexolytic secession (Seifert et al. 2011; Wendt et al. 2018). Xylocladium claviforme was first described as Masoniomyces claviformis J.L. Crane \& Dumont by Crane and Dumont (1975), on rotting wood, and later transferred to the genus Xylocladium by Arx (1982). In X. claviforme the conidiogenous cells have flattened scars at the point of origin of the conidia, whereas $X$. clautriavii has conidiogenous cells with 3-4 protruding scars, in addition to smaller conidia (5-6 $\times 3 \mu \mathrm{m})$ (Saccardo and Trotter 1913). The Brazilian material presented larger conidia $(6-8 \times 2.5-3$ vs. $4-7 \times 2 \mu \mathrm{m})$ than those described by Crane and Dumont (1975) and Heredia et al. (2004). This is the first record of this species for South America.

\section{DISCUSSION}

This is the first study dealing with conidial fungi associated with decomposing leaf litter of Cedrela odorata in Brazil. Until now, only a leaf spot was registered in this tree species, caused by Pseudobeltrania cedrelae (Hanada et al. 2005). This agent of leaf spot was not found in the present study.

Among the listed species, 51 species have already been found in Pará state and other Brazilian states, on several substrates. Previous records of conidial fungi in the state of Pará were known for the localities of Capitão-Poço (Pfenning 1997), Melgaço (Hernández-Gutiérrez et al. 2009; Monteiro et al. 2010; Hernández-Gutiérrez 2013), Santa Bárbara (CRIA 2017) and Belém (Rodrigues 1994; Castro et al. 2011; 2012; CRIA 2017). Our results increase the known number of conidial fungi species in Pará to 383, being the state of northern Brazil with the highest known diversity of this group.

Accordingly, our results increase the known diversity of conidial fungi in the municipality of Belém. In APA Combu Island there was an increment from 253 known species (Rodrigues 1994; Castro et al. 2011; 2012; CRIA 2017) to 278 species. This was the second record of conidial fungi in the Zoobotanical Park of Museu Goeldi, after the pioneering study by Paul C. Hennings in the early 1900s (Batista et al. 1966). This was the first record of conidial fungi for Bosque Rodrigues Alves Botanical Garden.

Among the new records found, Acarocybiopsis cubitaensis, $D$. biguttulata and $X$. claviforme have so far been recorded only in tropical environments, while C. abramovii presents a wide distribution, with records in several countries of temperate and tropical regions.

\section{CONCLUSIONS}

This study contributed to the knowledge about conidial fungi associated with Cedrela odorata, and to the expansion of the geographic distribution of several conidial fungi in the tropical region, including 46 new additions for the Brazilian Amazon, and new records for South America and the American continent. Our data evidences the need to expand collection efforts in less explored areas in the Brazilian Amazon.

\section{ACKNOWLEDGMENTS}

The first author thanks Conselho Nacional de Desenvolvimento Científico e Tecnológico (CNPQ) for a master's scholarship. We are grateful to the Museu Paraense Emílio Goeldi (MPEG) and Universidade Federal Rural da Amazônia (UFRA) for the infrastructure and support given to this research. We also thank Laboratório de Micologia (Universidade Estadual de Feira de Santana) for the support offered in the identification stage, and parataxonomist M.R. Santos for the identification of plants and support in the field.

\section{REFERENCES}

Albernaz, A.L.K.M.; Avila-Pires, T.C.S. 2009. Espécies ameaçadas de extinção e áreas críticas para a biodiversidade no Pará. Museu Paraense Emílio Goeldi, Belém, 60p.

Arx, J.A. 1982. The genus Dicyma, its synonyms and related fungi. Proceedings van de Koninklijke Nederlandse Akademie van Wetenschappen Section C, 85: 21-28.

Batista, A.C.; Bezerra, J.L.; Peres, G.E.P. 1964. Alguns Phyllachora Nits. do Pará e do Amazonas. Publicação do Instituto de Micologia da Universidade do Recife \& Instituto Nacional de Pesquisas da Amazônia, Conselho Nacional de Pesquisas, 437: 1-14.

Batista, A.C.; Falcão, R.G.S.; Peres, G.E.P.; Moura, N.R. 1966. Fungi Paraenses (Revisão da Coleção de Paul C. Hennings do Museu Paraense Emílio Goeldi). Publicação do Instituto de Micologia, 506: 10-290.

Cai, L.; Zhang, K.; McKenzie, E.H.C.; Hyde, K.D. 2003. Freshwater fungi from bamboo and wood submerged in the Liput River in the Philippines. Fungal Diversity, 13: 1-12.

Carmo, L.T.; Monteiro, J.S.; Gusmão, L.F.P.; Sotão, H.M.P.; Gutiérrez, A.H.; Castañeda-Ruiz, R.F. 2014. Anabahusakala, a new genus from the Brazilian Amazon rainforest. Mycotaxon, 127: 11-15.

Carmo, L.T.; Sotão, H.M.P.; Brito, F.M.; Moura, M.F.; Oliveira, J.R. 2016. Riqueza de fungos causadores de ferrugens em plantas hospedeiras da Região Metropolitana de Belém, PA, Brasil. Hoehnea, 43: 557-573.

Castañeda-Ruiz, R.F.; Heredia, G.; Gusmão, L.F.P.; Li, D.W. 2016. Fungal diversity of Central and South America. In: Li, D.W. (Ed.) Biology of Microfungi. Springer International Publishing, Switzerland, p.197-217.

Castro, C.C.; Gutiérrez, A.H.; Sotão, H.M.P. 2011. Novos registros de fungos anamorfos (hifomicetos) para o Neotrópico e América do Sul. Brazilian Journal of Botany, 34: 515-521. 
Castro, C.C.; Gutierrez, A.H.; Sotão, H.M.P. 2012. Fungos conidiais em Euterpe oleracea Mart. (Açaizeiro) na Ilha do Combu, ParáBrasil. Acta Botanica Brasilica, 26: 761-771.

Cazau, M.C.; Arambarri, A.M.; Cabello, M.N. 1990. New hyphomycetes from Santiago river. IV. (Buenos Aires Province, Argentina). Mycotaxon, 38: 21-25.

Crane, J.L.; Dumont, K.P. 1975. Hyphomycetes from the West Indies and Venezuela. Canadian Journal of Botany, 53: 843-851.

CRIA (Centro de Referência e Informação Ambiental), 2017. Specieslink - simple search (http://www.splink.org.br/index). Accessed on 01/06/2017.

Delgado, G.; Koukol, O. 2016. Microfungi from Nicaragua in a historical collection kept at the Herbarium of the Charles University in Prague. Cryptogamie Mycologie, 37: 15-36.

Dix, N.J.; Webster, J. 1995. Fungal Ecology. Chapman \& Hall, London, $549 \mathrm{p}$.

Ellis, M.B. 1971. Dematiaceous Hyphomycetes. Commonwealth Mycological Institute, Kew, 608p.

Engler, A.; Prantl, K. 1900. Die natürlichen Pflanzenfamilien nebst ihren Gattungen und wichtigeren Arten insbesondere den Nutzpflanzen. W. Engelmann, Leipzig, 570p.

Farr, D.F; Rossman, A.Y. 2017. Fungal Databases, Systematic Mycology and Microbiology Laboratory, ARS, USDA. (http:// nt.ars-grin.gov/fungaldatabases/). Accessed on 5/06/2017.

Ferreira, L.V.; Muñoz, S.H.; Parolin, P.; Chaves, P.P. 2012. O efeito da fragmentação e isolamento florestal das áreas verdes da região metropolitana de Belém. Pesquisas, Botânica, 63: 357-367.

Flora do Brasil 2020 em construção, 2017. Jardim Botânico do Rio de Janeiro (http://reflora.jbrj.gov.br/reflora/floradobrasil/FB25). Accessed on $02 / 06 / 2017$.

Forzza, R.C.; Filardi, F.L.R.; Costa, A.; Carvalho-Júnior, A.A.; Peixoto, A.L.; Walter, B.M.T.; et al. 2010. Catálogo de plantas e fungos do Brasil. Vol. 1. Andrea Jakobsson Estúdio, Instituto de Pesquisas do Jardim Botânico do Rio de Janeiro, Rio de Janeiro, 875p.

Goh, T.K.; Hyde, K.D. 1997. A revision of Dactylaria, with description of $D$. tunicata sp. nov. from submerged wood in Australia. Mycological Research, 101: 1265-1272.

Goh, T.K.; Hyde, K.D. 1999. Fungi on submerged wood and bamboo in the Plover Cove Reservoir, Hong Kong. Fungal Diversity, 3: 57-85.

Gusmão, L.F.P.; Grandi, R.A.P. 1997. Hyphomycetes com conidioma dos tipos esporodóquio e sinema associados a folhas de Cedrela fissilis (Meliaceae), em Maringá, PR, Brasil. Acta Botanica Brasilica, 11: 123-134.

Hanada, R.E.; Gasparotto, L.; Ferreira, F.A. 2005. Primeiro relato de mancha foliar em Cedrela odorata causada por Pseudobeltrania cedrelae. Fitopatologia Brasileira, 30: 299-301.

Hennings, P. 1900. Fungi Paraenses I. Hedwigia, 39: 76-80.

Hennings, P. 1902. Fungi S. Paulenses II. a cl. Puttemans collecti. Hedwigia, 41: 295-311.

Heredia, G.; Estebanez, M.R.; Arias, R.M.; Mena-Portales, J.; Mercado-Sierra, A. 2004. Adiciones al conocimiento de la diversidad de los hongos conidiales del bosque mesófilo de montańa del estado de Veracruz. Acta Botanica Mexicana, 66: 1-22.
Heredia, G.A.; Mota, R.M.A.; Mena-Portales, J.; Mercado-Sierra, A. 2006. Adiciones al conocimiento de la diversidad de los hongos conidiales del bosque mesófilo de montaña del estado de Veracruz II. Acta Botanica Mexicana, 77: 15-30.

Hernández-Gutiérrez, A.; Monteiro, J.S.; Sotão, H.M.P. 2009. Hifomicetos (fungos anamorfos) associados a palmeiras na Floresta Nacional de Caxiuanã, PA, Brasil. In: Lisboa, P. (Org.). Caxiuanã: Desafios para a conservação de uma floresta nacional na Amazônia. Museu Paraense Emílio Goeldi, Belém, p.397-405.

Hernández-Gutiérrez, A. 2013. New or rare fungi from eastern Amazonia. 1. Circinoconiopsis amazonica gen. and sp. nov. Mycotaxon, 123: 107-111.

Hernández-Restrepo, M.; Gené, J.; Mena-Portales, J.; Cano, J.; Madrid, H.; Castañeda-Ruiz, R.F.; Guarro, J. 2014. New species of Cordana and epitypification of the genus. Mycologia, 106: 723-734.

IUCN Red List of Threatened Species, Version 2017-1 (http://www. iucnredlist.org/). Accessed on 15/06/2017.

Matsushima, T. 1975. Icones Microfungorum a Matsushima Lectorum. Published by the author, Kobe, 209p.

Mena-Portales, J.; Hernández-Gutiérrez, A.; Mercado-Sierra, A. 1999. Acarocybiopsis, a new genus of synnematous hyphomycetes from Cuba. Mycological Research, 103: 1032-1034.

Monteiro, J.S.; Gutierrez, A.H.; Sotão, H.M.P. 2010. Fungos anamorfos (Hyphomycetes) da Floresta Nacional de Caxiuanã, Pará, Brasil: novos registros para o Neotrópico. Acta Botanica Brasilica, 24: 871-876.

Mycobank Database, 2017. (http://www.mycobank.org). Accessed on $02 / 07 / 2017$.

Paulus, B.; Gadek, P.; Hyde, K.D. 2003. Two new species of Dactylaria (anamorphic fungi) and an update of species in Dactylaria sensu lato. Fungal Diversity, 14: 143-156.

Pfenning, L.H. 1997. Soil and rhizosphere microfungi from Brazilian tropical forest ecosystems. In: Hyde, K.D. (Org.). Diversity of tropical microfungi. Hong Kong University Press, Hong Kong, p.337-362.

Preuss, C.G.T. 1851. Übersicht untersuchter Pilze, besonders aus der Umgegend von Hoyerswerda. Linnaea, 24: 99-153.

Raja, H.A.; Stchigel, A.M.; Miller, A.N.; Crane, J.L.; Shearer, C.A. 2007. Hyphomycetes from the Great Smoky Mountains National Park, including three new species. Fungal Diversity, 26: 271-286.

Rao, N.K.; Agarwal, D.K.; Kunwar, I.K. 2005. Manoharachariomyces, a new hyphomycetous fungus from India. Indian Phytopathology, 58: 96-99.

Rodrigues, K. 1994. The foliar fungal endophytes of the Amazonian palm Euterpe oleracea. Mycologia, 86: 376-385.

Saccardo, P.A. 1880. Conspectus generum fungorum Italiae inferiorum nempe ad Sphaeropsideas, Melanconieas et Hyphomyceteas pertinentium systemate sporologico dispositorum. Michelia, 2: 1-38.

Saccardo, P.A.; Trotter, A. 1913. Supplementum Universale, Pars IX. Sylloge Fungorum, 22: 1-1612. 
Seifert, K.; Morgan-Jones, G.; Gams, W.; Kendrick, B. 2011. The Genera of Hyphomycetes. CBS Biodiversity Series no. 9. CBSKNAW Fungal Biodiversity Centre, Utrecht, 997p.

Seman, E.O.; Davydkina, T.A. 1983. De genere Cordana Preuss in URSS. Novosti Sistematiki Nizshikh Rastenii, 20: 114-118.

Trappe, J.M.; Schenck, N.C. 1982. Taxonomy of fungi forming endomycorrhizae. In: Schenck, N.C. (Ed.). Methods and principles of mycorrizal research. The American Phytopathological Society, St. Paul, p.1-9.

Viana, F.M.P.; Sousa, J.A.; Freire, F.C.O.; Pieniz, V.; Correia, D.; Araújo, J.D.M. 2012. Ocorrência e controle da manchade-Phyllachora em cedro no Estado do Ceará. Embrapa Agroindústria Tropical, Comunicado técnico, 186p.
Wendt, L.; Sir, E.B.; Kuhnert, E.; Heitkämper, S.; Lambert, C.; Hladki, A.I. et al. 2018. Resurrection and emendation of the Hypoxylaceae, recognised from a multigene phylogeny of the Xylariales. Mycological Progress, 17: 115-154.

Zelski, S.E.; Balto, J.A.; Do, C.; Raja, H.A.; Miller, N.A.; Shearer, C.A. 2014. Phylogeny and morphology of dematiaceous freshwater microfungi from Peru. IMA fungus, 5: 425-438.

RECEIVED: $01 / 12 / 2017$

ACCEPTED: 30/03/2018

ASSOCIATE EDITOR: Claudia Keller 